\title{
A Long-Term, Real-Life Parkinson Monitoring Database Combining Unscripted Objective and Subjective Recordings
}

\author{
Jeroen G. V. Habets ${ }^{1, *}$, Margot Heijmans ${ }^{1}$, Albert F. G. Leentjens ${ }^{2}{ }^{(}$, Claudia J. P. Simons $\left.^{2,3}{ }^{(}\right)$, Yasin Temel ${ }^{1}$, \\ Mark L. Kuijf ${ }^{4}$, Pieter L. Kubben ${ }^{1}$ (i) and Christian Herff ${ }^{1}{ }^{1}$
}

1 Department of Neurosurgery, School for Mental Health and Neurosciences, Faculty of Health, Medicine and Life Sciences, Maastricht University, 6229 ER Maastricht, The Netherlands; m.heijmans@maastrichtuniversity.nl (M.H.); y.temel@maastrichtuniversity.nl (Y.T.); p.kubben@mumc.nl (P.L.K.); c.herff@maastrichtuniversity.nl (C.H.)

2 Department of Psychiatry and Neuropsychology, School for Mental Health and Neurosciences, Faculty of Health, Medicine and Life Sciences, 6229 ER Maastricht, The Netherlands; a.leentjens@maastrichtuniversity.nl (A.F.G.L.); c.simons@maastrichtuniversity.nl (C.J.P.S.)

3 GGzE (Mental Healthcare Eindhoven), 5626 ND Eindhoven, The Netherlands

4 Department of Neurology, School for Mental Health and Neurosciences, Faculty of Health, Medicine and Life Sciences, Maastricht University, 6229 ER Maastricht, The Netherlands; mark.kuij@mumc.nl

* Correspondence: j.habets@maastrichtuniversity.nl

Citation: Habets, J.G.V.; Heijmans, M.; Leentjens, A.F.G.; Simons, C.J.P.; Temel, Y.; Kuijf, M.L.; Kubben, P.L.; Herff, C. A Long-Term, Real-Life Parkinson Monitoring Database Combining Unscripted Objective and Subjective Recordings. Data 2021, 6, 22. https://doi.org/10.3390/ data6020022

\section{Academic Editors:}

Joaquín Torres-Sospedra and Aleksandr Ometov

Received: 11 February 2021

Accepted: 18 February 2021

Published: 23 February 2021

Publisher's Note: MDPI stays neutral with regard to jurisdictional claims in published maps and institutional affiliations.

Copyright: (c) 2021 by the authors. Licensee MDPI, Basel, Switzerland. This article is an open access article distributed under the terms and conditions of the Creative Commons Attribution (CC BY) license (https:/ / creativecommons.org/licenses/by/ $4.0 /)$.

\begin{abstract}
Accurate real-life monitoring of motor and non-motor symptoms is a challenge in Parkinson's disease (PD). The unobtrusive capturing of symptoms and their naturalistic fluctuations within or between days can improve evaluation and titration of therapy. First-generation commercial PD motion sensors are promising to augment clinical decision-making in general neurological consultation, but concerns remain regarding their short-term validity, and long-term real-life usability. In addition, tools monitoring real-life subjective experiences of motor and non-motor symptoms are lacking. The dataset presented in this paper constitutes a combination of objective kinematic data and subjective experiential data, recorded parallel to each other in a naturalistic, long-term real-life setting. The objective data consists of accelerometer and gyroscope data, and the subjective data consists of data from ecological momentary assessments. Twenty PD patients were monitored without daily life restrictions for fourteen consecutive days. The two types of data can be used to address hypotheses on naturalistic motor and/or non-motor symptomatology in PD.
\end{abstract}

Dataset: DataVerseNL: https:/ / doi.org/10.34894/5HHK8H

Dataset License: CC0_“Public Domain Dedication".

Keywords: Parkinson's disease; real-life; unscripted; naturalistic monitoring; wearable sensors; motor diaries; ecological momentary assessments; experience sampling method

\section{Summary}

Parkinson's disease (PD)'s world-wide prevalence is expected to double to over 12 million patients by 2040 [1]. Current treatment strategies are symptomatic, mainly focus on improving motor function, and start with oral dopaminergic replacement medication. Refractory motor symptoms, adverse effects, or (non-)motor fluctuations can indicate advanced treatments, such as continuous levodopa administration or deep brain stimulation, or additional non-dopaminergic therapies [2,3]. A challenge in PD care is to improve symptom monitoring during patients' real life, in between clinical visits. Continuous, passive PD monitoring is suggested to improve therapy evaluation and titration by decreasing reliability on patient recall, and limitations of current monitor tools such as the lack of unobtrusive, repetitive assessment $[4,5]$. Wearable sensors monitoring motor symptoms are probably the best-known example of passive monitoring [6-9]. Wearable motion 
sensors are also suggested to investigate non-motor symptoms such as depression [10]. Additionally or complementary to continuous objective monitoring, continuous subjective monitoring via electronic (e-)diaries is suggested to contribute to both motor and non-motor PD monitoring in real life [11,12].

Due to the ubiquitous presence of smartphones and smartwatches with dedicated mHealth-applications, the collection and analysis of real-life data has increased exponentially over the last decade. Most of these devices allow objective motion data collection via inertial measurement units (IMUs). IMUs typically contain accelerometers and/or gyroscopes. In addition to these objective measures, subjective information about the patient's status can be obtained through diary methods or regular questionnaires administered via mHealth-applications. A valid translation of these real-life, or naturalistic, data into clinically or scientifically relevant information is an important challenge for researchers involved in PD and many (neuro)psychological and somatic diseases [6,12].

The presented multi-modal data were collected to improve understanding of the feasibility, usability, and validity of real-life PD monitoring, with a focus on motor symptoms. Objective data were collected via bilateral wrist and a chest IMU containing accelerometers and gyroscopes. Subjective data were collected via smartphone-based ecological momentary assessments (EMA), also called experience sampling methods. The practical feasibility of this novel combined method in a general PD population was demonstrated before [11]. We showed a good completion of objective and subjective data collection, with an acceptable burden for PD patients, and without high variability in completion between or within days.

These naturalistic long-term objective and subjective data aim to overcome the lack of unobtrusive, momentary, repetitive assessments of currently available PD motor monitoring devices $[7,13,14]$. Substantial concerns exist about the first-generation PD monitor devices regarding real-life validity, and their specific intended role in clinical practice $[15,16]$. Defining the exact role in clinical practice of a clinically supportive, data-driven tool is of critical importance to realize successful and impactful implementation $[17,18]$. In our data, the subjective data complements the motor monitoring by the objective data. Combining continuous objective and subjective data can help to overcome the well-known challenge of translating scripted, lab-based monitor methods, to unscripted, real-life monitor methods $[19,20]$. More specifically, it can serve as an alternative, continuous gold standard informing about subjectively experienced PD symptomatology, parallel to naturalistic sensor data. Traditional PD monitor instruments, such as the Movement Disorders Society Unified Parkinson Disease Rating Scale (MDS-UPDRS) and the Parkinson Disease Quality of Life Questionnaire (PDQ-39) are limited as they require the physical presence of trained clinicians, one assessment covers days to weeks, and their usability for longitudinal follow-up has been questioned [21-23]. As a proof-of-concept, we successfully predicted subjective EMA-answers regarding tremor severity based on objective motion data in a single participant [24].

Non-motor symptoms are notoriously disregarded in PD management, despite their repeatedly demonstrated effect on patients' quality of life [25]. Dedicated non-motor symptom scales are increasingly available and applied; however, naturalistic momentary assessment is no standard practice yet. EMA is explored for psychiatric disorders such as depression [26], and evidence suggests a role of EMA in monitoring or treating depression [27]. EMA may contribute to clinical practice and scientific research regarding non-motor symptoms in PD [28]; this hypothesis requires, however, further investigation.

This paper and its accompanying database aim to enable and stimulate PD researchers to answer research questions regarding real-life motor and/or non-motor symptoms, using objective data, subjective data, or both. For the patients who reported to suffer from motor fluctuations, motor-related motion sensor analyses can be performed. The potential value of these data is underlined by previous naturalistic PD monitoring work calling for continuous data sets containing self-reported motor states and motion sensor data [29]. Especially, the repetitive subjective symptom experiences via EMA offer new possibilities in motor 
monitoring development. Where recent validation studies aim on performance over longer time periods $[9,30]$, the additional ESM facilitates analyses over shorter time windows. Moreover, the ESM offers a higher symptom resolution than merely ON-versus OFFmedication. Open-code efforts such as the BEAT-PD data challenge offer well-performing publicly available algorithms to differentiate between ON and OFF, and detecting tremors and dyskinesia [31]. These proposed signal processing and prediction analyses can be applied on finer-grain symptom resolutions in our dataset.

Further, many non-motor symptom-related research questions and hypotheses can be explored with these data. Non-motor symptoms are increasingly suggested to be part of continuous PD monitoring [12], and a concrete example is the suggestion to include patient-reported outcomes to monitor non-motor symptoms after deep brain stimulation for PD [32]. Research into general non-motor monitoring, as well as research into such specific non-motor monitoring applications, can profit from the data presented here.

With this data and this accompanying descriptor, we contribute to open-science for PD in general, and open-source, reproducible algorithms for real-life PD monitoring $[29,31,33,34]$. Furthermore, the applied methodology to combine momentary objective data with high-frequency objective data can be extrapolated to (EMA-)research in general.

\section{Data Description}

The data is publicly available via DataverseNL repository "EMA and wearable sensor monitoring in PD", under CC0—"Public Domain Dedication" license (https: / / doi.org/10.348 94/5HHK8H (accessed on 11 February 2021)) [35]. We provide raw sensor data and raw EMA data separately and unmerged, to enable every researcher to process the data as desired.

The code used for extracting, aligning, and merging both data types, including the example analysis, is available on https://github.com/jgvhabets/sensor_EMA_PD_ monitoring (accessed on 11 February 2021).

\subsection{Objective Sensor Data}

Sensor data are stored in European Data Format [36] (edf)-files, and organized on the DataVerseNL repository in separate patient folders. Each patient folder contains multiple edf-files representing all 14 recording days.

- The name of every edf-file contains first the sensor name, followed by the start date and time of the recording. For example, in folder "110001", the file "13792_20180828_0752223.edf" contains the recording from participant 110001 with sensor 13792, which started recording on 28 August 2018, at 07:52:23. The read-me file "READ_ME_EMA_SENSOR_PD.txt" explains which sensor numbers represent left wrist, right wrist, or chest IMUs. The sensors actively recorded when they were not connected to a USB-charging device.

- Each edf-file contains six channels (representing the $x_{-}, \mathrm{y}_{-}$, and $\mathrm{z}$-axes for, respectively, accelerometer and gyroscope), including timestamps. Acceleration is recorded in $\mathrm{m} / \mathrm{s}$ per second, and rotation is recorded in degrees per second.

- Prior to the first recording day, the clocks of all three sensors were reset and synchronized. The manufacturer assures temporal drift to be negligible over the period of two weeks with respect to merging and pairing with EMA-assessments.

- $\quad$ Single edf-files were created when a sensor was disconnected from the charger. A file continued storing data until the sensor was connected to a charger again and the file closed. If a recording passed midnight (00:00:00), the file closed as well, and a new file was created and continued storing data.

\subsection{Subjective EMA Data}

\subsubsection{EMA Data Organization}

The EMA method consisted of three types of questionnaires: a "beep" questionnaire, a daily morning questionnaire, and a daily evening questionnaire (Table 1). The "beep" questionnaire was identically offered seven times per day, with an accompanying notification; the morning questionnaire was available to complete on own initiative between 
06:00 and 12:00; and the evening questionnaire was available to complete on own initiative between 20:00 and 03:00.

- The EMA data from all patients are stored in "EMA_data.csv".

- The fist column provides a patient number, corresponding to the sensor data folder names.

- Then, two columns provide timestamps indicating the start time and end time of beep-questionnaire completion.

- These are followed by columns providing the answers on the items from the beepquestionnaire.

- Then, columns provide the answers on the morning and evening questionnaires from the corresponding day.

- The file "EMA_data_coding.xlsx" provides a clear explanation of the coding of all questionnaire items and answers.

\subsubsection{EMA Content}

If no multiple-choice is provided in square brackets, items are answered on a Likert scale from 1 to 7 .

Table 1. Content of Parkinson's-specific experience sampling questionnaires.

\begin{tabular}{|c|c|}
\hline Beep Questionnaire (Repetitive, Seven Times Daily) & \\
\hline Affect and cognitive items & Possible answers \\
\hline $\begin{array}{l}\text { I feel well, down, fearful, stressed, sleepy, tired, cheerful, relaxed } \\
\text { (eight different items) }\end{array}$ & 7-point Likert scale (eight times) \\
\hline I can concentrate well & 7-point Likert scale \\
\hline I experience hallucinations & 7-point Likert scale \\
\hline \multicolumn{2}{|l|}{ Contextual items } \\
\hline I am at... & (home, work, travelling, family/friend's place, in public) \\
\hline I am with ... & $\begin{array}{l}\text { (nobody, family, partner, colleagues, friends) (multiple choice, } \\
\text { multiple items could be selected) }\end{array}$ \\
\hline I am doing ... & $\begin{array}{l}\text { (work, resting, household/odd jobs, sports, something else) } \\
\text { (multiple choice, multiple items could be selected) }\end{array}$ \\
\hline \multicolumn{2}{|l|}{ Physical items } \\
\hline I can do this (my current activity) without hinder & 7-point Likert scale \\
\hline I am comfortable walking and standing & 7-point Likert scale \\
\hline I can sit or stand still easily & 7-point Likert scale \\
\hline I can speak easily & 7-point Likert scale \\
\hline I can walk easily & 7-point Likert scale \\
\hline I experience tremor & 7-point Likert scale \\
\hline I am moving slow & 7-point Likert scale \\
\hline I experience stiffness & 7-point Likert scale \\
\hline My muscles are tensioned & 7-point Likert scale \\
\hline I am uncontrollable moving & 7-point Likert scale \\
\hline \multicolumn{2}{|l|}{ Dopaminergic medication items } \\
\hline I feel ... (regarding medication status) & [1: OFF, 2: ON $\rightarrow$ OFF, 3: ON, 4: OFF $\rightarrow$ ON] \\
\hline I took Parkinson's medication since last beep & (yes, no, I do not recall) \\
\hline
\end{tabular}


Table 1. Cont.

\begin{tabular}{cc}
\hline Beep Questionnaire (Repetitive, Seven Times Daily) & \\
\hline Morning questionnaire & 7-point Likert scale \\
\hline I slept well & 7-point Likert scale \\
\hline I woke up often last night & 7-point Likert scale \\
\hline I feel rested & 7-point Likert scale \\
\hline It was physically difficult to get up & 7-point Likert scale \\
\hline It was mentally difficult to get up & 7-point Likert scale \\
\hline Evening questionnaire & 7-point Likert scale \\
\hline I had long OFF periods today & 7-point Likert scale (five times) \\
\hline I had many OFF periods today & 7-point Likert scale \\
\hline Walking, dressing, eating/drinking, personal care, household activities \\
went well today (five separate items)
\end{tabular}

\section{Methods}

\subsection{Participants}

Twenty idiopathic PD patients participated in this study (Table 2). Patients were recruited via their treating neurologist or neurosurgeon at Maastricht University Medical Centre. Inclusion criteria were diagnoses of idiopathic PD, age between 18 and 80 years, in possession of a smartphone (minimal iOS 8 or Android 4), mastering spoken and written Dutch language, and available during two consecutive weeks of representative daily activities (meaning no holidays or planned hospital admission). No participants were excluded because of cognitive deficits (less than 24 points on the Montreal Cognitive Assessment). Hoehn and Yahr scores and levodopa equivalent daily dosages were collected. Participants 110002, 110003, 110004, 110006, 110008, 110014, 110016, 110017, 110018, 110019, 110020, and 110021 reported to suffer motor fluctuations despite dopaminergic (and deep brain stimulation) therapy. Disease-specific details are not included in the publicly available data set due to local ethical privacy regulations. Researchers can contact the authors when these data are requested.

Table 2. Demographics of included Parkinson's population.

\begin{tabular}{cc}
\hline Variable & Mean (Standard Deviation) or Proportion $(n)$ \\
\hline Gender $(n$ female $/ n$ male $)$ & $4 / 16$ \\
\hline Age (years) & $63(7)$ \\
\hline Levodopa Equivalent Daily Dosage $(\mathrm{mg})$ & $770(394)$ \\
\hline Hoehn and Yahr Scale $(n)$ & 2 \\
\hline 1 & 2 \\
\hline 1.5 & 7 \\
\hline 2 & 3 \\
\hline 2.5 & 3 \\
\hline 3 & 0 \\
\hline 3.5 & 1 \\
\hline 4 & $12 / 8$ \\
\hline Presence Motor Fluctuations $(n$ yes $/ n$ no $)$ & \\
\hline
\end{tabular}


This study was approved by the local medical ethical committee and written informed consent was obtained from all participants, including the approval to share the anonymized data (METC azM/UM 2017-0307).

\subsection{Study Design}

Participants were monitored via wearable sensors and EMA for 14 consecutive days and were instructed to not adjust their daily life routines or activities (Figure 1). An introductory meeting was held prior to day 1 at the patient's home during which the sensors were provided and the EMA application was installed and explained. A phone call consultation was performed at day 2 and day 8 to evaluate the progress and answer possible questions. After day 14, a researcher collected the sensors and evaluated the study period.

\subsection{Parkinson's-Specific EMA Method}

EMA is a validated method for observational studies or therapy evaluation in psychiatric and psychological diseases [37-39]. Subjective experiences are collected through questionnaires several times daily on semi-randomized moments. Recall-bias is minimized by allowing the patient to complete the questionnaire only during a short time window after notification, and by asking the patient to report his/her experience "at this moment". These repeated measurements aim to capture symptom fluctuations over the day, as well as slower fluctuating trends over longer time spans. The practical feasibility of this method in these 20 PD patients is described earlier [11].

We applied a PD-specific EMA questionnaire in this dataset, containing affect, motor, and non-motor symptomatology, as well as contextual items. The morning questionnaire contains mainly items regarding sleep and fitness after waking, and the evening questionnaire covers mainly motor functioning and symptomatology during the day, to enrich analyses on day levels. The development and internal validation of this PD EMAquestionnaire is extensively described earlier [40].

EMA and eDiaries are thought to be more sustainable tools to capture subjective intraday PD fluctuations in the future than paper diaries, which are more limited by procrastination, recall bias, and diary fatigue [41,42].

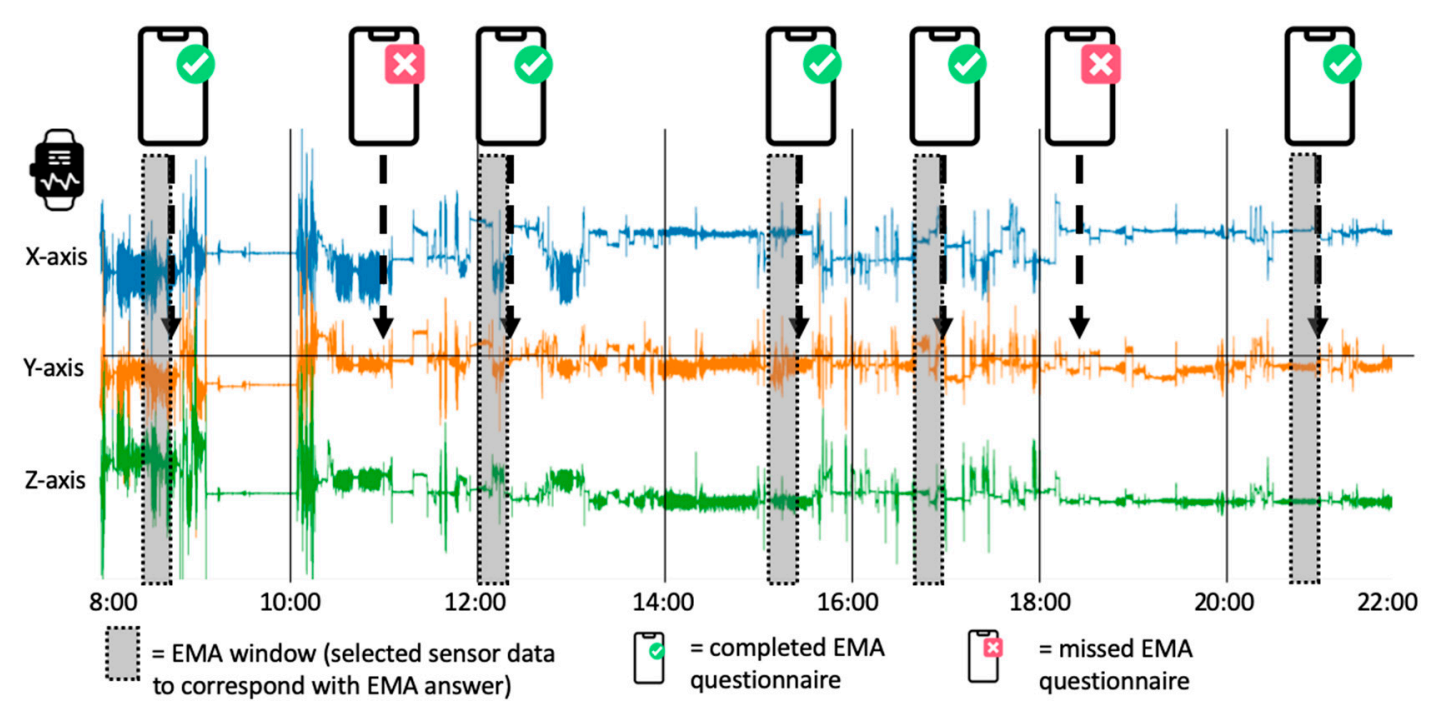

Figure 1. Schematic overview of one day of data collection. The blue, orange, and green lines represent, respectively, the signal on the $\mathrm{x}_{-}^{-}, \mathrm{y}^{-}$, and $\mathrm{z}$-axis of an accelerometer, or a gyroscope. The signals are shown between 8:00 a.m. and 10:00 p.m. Selected time spans of sensor data which will be compared with the corresponding ecological momentary assessment (EMA) answers are shown in grey. 


\subsection{Devices}

\subsubsection{PsyMate (EMA Application)}

The EMA monitoring was executed via a smartphone-based EMA application (PsyMate ${ }^{\circledR}$, Maastricht, The Netherlands). The application presented the beep questionnaire seven times daily on semi-randomized moments, one questionnaire per two-hour window between 08:00 and 22:00 (see Figure 1). In order to reduce recall bias, the participants had to start answering the questionnaire within $15 \mathrm{~min}$ after notification. After these $15 \mathrm{~min}$ the questionnaire could not be opened anymore and was labeled as missed. The participants were instructed to complete as many questionnaires as possible without adjusting their lifestyle and activities. They were asked to complete the morning and evening questionnaire every day. These questionnaires were available during the above-mentioned time spans but were not presented with a notification.

The answers to completed beep questionnaires, as well as to the morning and evening questionnaires, were automatically uploaded to a server provided by the application developer. Missed questionnaires were not registered in the database.

\subsubsection{MOX-5 (Wearable Sensor)}

The patients used three wearable sensors, one located at each wrist and one at the sternum attached to a necklace (MOX5, Maastricht Instruments $\odot$, Maastricht, The Netherlands). The six degrees-of-freedom wearable sensors contained a tri-axial accelerometer and a tri-axial gyroscope, and recorded unprocessed raw data. The axial orientation of the wrist sensors was the following: $x$ was parallel to the arm length, $y$ recorded sideways movement in anatomical position, and $\mathrm{z}$ recorded front/back movement in anatomical position. The axial orientation of the chest sensor was the following: $x$ was parallel to the body length, $y$ recorded movement sideways, and $\mathrm{z}$ recorded movement to the front or back. The accelerometer covered an amplitude range of $\pm 8 \mathrm{~g}$ and the gyroscope covered a range of \pm 2000 degrees/s. Data were collected with a sampling rate of $200 \mathrm{~Hz}$. The sensors stored all data on a built-in memory disk and did not automatically transfer data or provide real-time assessments. The participants were instructed to wear the sensors from the moment they rose in the morning, until they went to bed at night (ideally at least between 08:00 until 22:00), except during showering or bathing.

\section{User Notes}

\subsection{Software}

We performed our data pre-processing and analysis in Jupyter Notebooks for Python (Python version 3.6, Project Jupyter@, https:/ /jupyter.org (accessed on 11 February 2021), revision fe7c2909). We used software packages: pyedflib, pandas (version 0.24.2) [43], Numpy (version 1.16.4) [44], and scikit-learn (version 0.21.2) [45].

\subsection{Interpretation of Data Quantity and Quality}

Detailed descriptions of these results are reported earlier [11,40]. Most of the participants experienced the data collection as not incriminating (17 out of $20.85 \%$ ), and $90 \%$ (18 out of 20) did not adapt their daily activities. On average, the participants wore the wearable sensors $94 \%$ of the instructed wearing time, resulting in almost $15 \mathrm{~h}$ of sensor data collected daily. EMA completion rates for beep, morning, and evening questionnaires were, respectively, $79 \%, 97 \%$, and $94 \%$. No differences were seen in completion between different study days, or different daily moments. For three participants $(110007,110010$, and 110015), more than $25 \%$ of the sensor data corresponding to the EMA beeps were missing due to practical data storage issues.

Internal validity of the EMA answers is explored by correlating subjectively reported concepts as positive versus negative affect, motor symptom severity, motor functioning, and dopaminergic medication states with each other. Positive correlations between positive affect and motor functioning and less symptom severity were hypothesized. Additionally, dopaminergic off-medication states were hypothesized to correlate positively with motor 
symptom severity, and to correlate negatively with motor functioning and positive affect. The evening questionnaire on motor functioning and motor symptoms during the past day were hypothesized to correlate in the same way as described above. On a group level, this hypothesis was confirmed by correlations linking positive affect, with fewer motor symptoms, better motor functionality, and on-medication states, and vice versa. On an individual level, not enough fluctuations were captured for every individual participant to reproduce this. Seventy-six percent of EMA beeps were answered in dopaminergic on-state, $21.5 \%$ in transition state between on- and off-state, and $2.5 \%$ in off-state. Mild fluctuations are therefore more likely to be captured more often in this data than severe fluctuations. Due to the high overall completion rates, and in-person evaluations with the participants, we suggest the low number of off-states can be explained by a low prevalence of true off-states. For medication-state analysis, it therefore can be suggested to compare on-state versus non-on-state (transition on/off plus off-state).

\subsection{Combined Data Processing and Analyzing: Practical Example of Dopaminergic Fluctuation Detection}

As an example, we propose a pipeline to align and merge two data types with varying sample frequencies. In this process, we decided to select sensor data from the $15 \mathrm{~min}$ preceding the start of EMA beep questionnaire completion. These $15 \mathrm{~min}$ are chosen because the patients were instructed to complete the questionnaire according to their experiences at that exact moment ("How do you feel at this moment"). The 15 min block was a pragmatic and arbitrary decision; researchers might deviate from this approach based on their own arguments or hypothesis.

To support the usability of the data, we will give an example of dopaminergic fluctuation detection in participant 110018, suffering from strong symptom fluctuations [24]. We will merge both data types, extract features from the sensor data, and present correlation between the objective and subjective data by predicting the subjective reported medication state based on the objective sensor features. We extract features designed for wrist-sensor data, and will repeat classification analyses based on wrist- and chest-sensor data. The wrist-sensor analysis is comparable to an analysis in previous work [11]. Here, we repeat this analysis with data from the chest-sensor. Although the wrist-data and the chestdata were recorded during the same time points, we hypothesize that the classification analysis based on wrist-data is more successful to differentiate medication-state.

\subsubsection{Data Merging}

To enable merging of the EMA data and the sensor data, the completed beep questionnaires from the EMA data were used as a reference frame. For sensor data extraction, the timestamps of all starting moments of completed beep questionnaires were extracted and the raw sensor data corresponding to each 15-min block preceding these EMA time stamps were selected. Raw sensor data were first down-sampled to $100 \mathrm{~Hz}$ since a higher sample rate was not necessary for the intended analysis. This resulted in a data frame containing 90,000 rows of sensor data $(15 \mathrm{~min}$ of $100 \mathrm{~Hz}$ ) corresponding to every complete beep questionnaire. Each row consisted of three values ( $\mathrm{x}-, \mathrm{y}-, \mathrm{z}$-axis) for each of the three accelerometers and the gyroscopes.

\subsubsection{Sensor Data Pre-Processing and Feature Extraction}

Features representing the research topic of interest, in this case, dopaminergic medication fluctuation, have to be extracted from the raw, $100 \mathrm{~Hz}$ sensor data. Depending on the features and hypothesis, researchers can be interested in feature values per, e.g., $60 \mathrm{~s}$ of extracted sensor data. This time span is referred to as the feature window length. We extracted various features designed for wrist-accelerometer and wrist-gyroscope data representing bradykinesia or medication fluctuation [8]. By calculating features, a new dataset is created, in which each row contained the timestamp and the feature value for one feature window length. 
For this practical example provided, we applied a feature window length of $900 \mathrm{~s}$ [24], and did not apply overlapping epochs in the sensor data. The following temporal domain features were extracted: the root mean square (RMS) of every accelerometer-axis feature window length, and the amplitude range, representing the range between the minimal and maximum amplitude per accelerometer-axis feature window length. The following spectral domain features were extracted: the spectral power density for frequencies corresponding to tremors $(3.5-7.5 \mathrm{~Hz})$ for both accelerometer-axes and gyroscope-axes, and the spectral power density for frequencies corresponding to bradykinesia $(0.5-3 \mathrm{~Hz})$ per accelerometeraxis, the dominant frequency per accelerometer-axis, and the dominant energy ratio per accelerometer-axis (dividing the energy within the dominant frequency by the total sum of the energy in all frequencies).

\subsubsection{Classification Analysis}

The corresponding EMA items on medication status were used as binary labels for the extracted sensor data. We trained a logistic regression model in a 5-fold cross-validation. Applied on wrist-worn sensor data, the classifier differentiated between the medication states with an area under the receiver operator curve (AUROC) of 0.73 (Figure 2A). Applied on chest-worn sensor data, the same classifier was not able to discriminate between the medication states above chance level (AUROC of 0.51) (Figure 2B).

The presented examples show that subjectively reported experiences of medication fluctuation can be predicted from features derived from the motion sensor data.
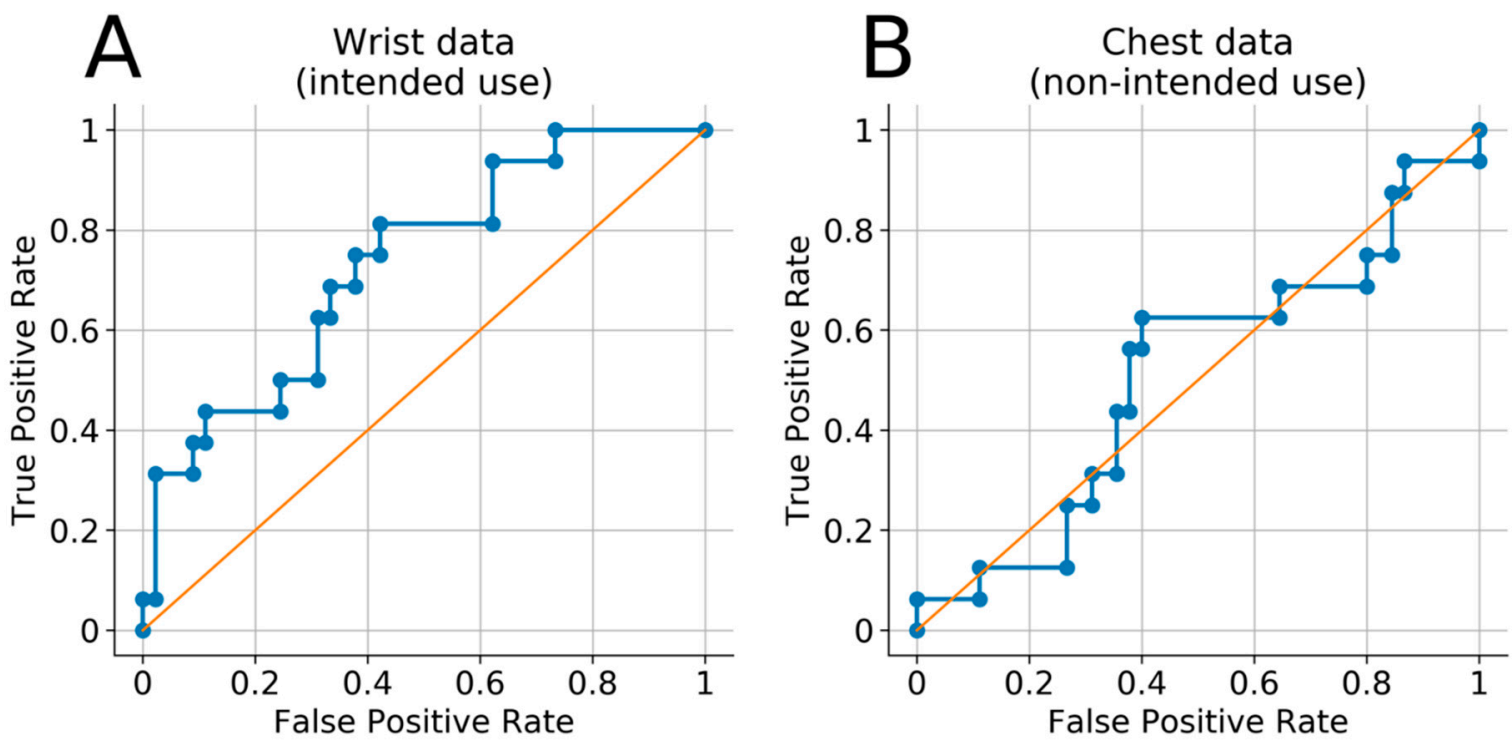

Figure 2. Receiver Operator Curves (ROC) for differentiating medication-state of a patient with symptom fluctuations based on sensor features which are developed for wrist-worn sensor data. (A) ROC for analysis with features extracted from wrist-worn data. (B) ROC for analysis with features extracted from chest-worn data. Figure 2A is based on comparable analyses as in our feasibility study [11].

Author Contributions: Conceptualization, J.G.V.H., M.H., C.J.P.S., A.F.G.L., Y.T., M.L.K., P.L.K.; methodology, all; software, J.G.V.H., C.H.; validation, J.G.V.H., M.H., C.H.; formal analysis, J.G.V.H., M.H., C.H.; resources, P.L.K., Y.T.; data curation, J.G.V.H.; writing-original draft preparation, J.G.V.H., C.H.; writing—review and editing, all; visualization, J.G.V.H.; supervision, C.H.; project administration, J.G.V.H., M.H.; funding acquisition, P.L.K., Y.T. All authors have read and agreed to the published version of the manuscript.

Funding: This research was funded by the Weijerhorst Foundation, UTAP Grant. 
Institutional Review Board Statement: This study was conducted according to the guidelines of the Declaration of Helsinki, and approved by the Institutional Review Board (Medical Ethical Testing Committee) azM/UM, file number 2017-0307.

Informed Consent Statement: Informed consent was obtained from all subjects involved in the study.

Data Availability Statement: Data is fully available at DataVerseNL: https:/ / doi.org/10.34894/5 HHK8H (accessed on 11 February 2021). The code used for extracting, aligned, and merging both data types, including the example analysis for the Technical Validation section, is available on https://github.com/jgvhabets/sensor_EMA_PD_monitoring (accessed on 11 February 2021).

Acknowledgments: We want to thank the ESM Expert Group of Maastricht University/Open University Heerlen for their advice on the design of the EMA methodology. We want to thank Karel Borkelmans for the development and technical support on the PsyMate application. We want to thank the clinical multidisciplinary Deep Brain Stimulation/ Movement Disorders team of Maastricht $\mathrm{UMC}+$ for advice during the design of the study.

Conflicts of Interest: The authors declare no conflict of interest.

\section{References}

1. Dorsey, E.R.; Sherer, T.; Okun, M.S.; Bloem, B.R. The Emerging Evidence of the Parkinson Pandemic. J. Parkinsons Dis. 2018, 8, 3-8. [CrossRef]

2. Odin, P.; Ray Chaudhuri, K.; Slevin, J.T.; Volkmann, J.; Dietrichs, E.; Martinez-Martin, P.; Krauss, J.K.; Henriksen, T.; Katzenschlager, R.; Antonini, A.; et al. Collective physician perspectives on non-oral medication approaches for the management of clinically relevant unresolved issues in Parkinson's disease: Consensus from an international survey and discussion program. Parkinsonism Relat. Disord. 2015, 21, 1133-1144. [CrossRef] [PubMed]

3. Seppi, K.; Weintraub, D.; Coelho, M.; Perez-Lloret, S.; Fox, S.H.; Katzenschlager, R.; Hametner, E.M.; Poewe, W.; Rascol, O.; Goetz, C.G.; et al. The Movement Disorder Society Evidence-Based Medicine Review Update: Treatments for the non-motor symptoms of Parkinson's disease. Mov. Disord. 2011, 26, 42-80. [CrossRef]

4. Papapetropoulos, S.; Mitsi, G.; Espay, A.J. Digital Health Revolution: Is it Time for Affordable Remote Monitoring for Parkinson's Disease? Front. Neurol. 2015, 6. [CrossRef] [PubMed]

5. Hansen, C.; Sanchez-Ferro, A.; Maetzler, W. How Mobile Health Technology and Electronic Health Records Will Change Care of Patients with Parkinson's Disease. J. Parkinsons Dis. 2018, 8, 41-45. [CrossRef] [PubMed]

6. Odin, P.; Chaudhuri, K.R.; Volkmann, J.; Antonini, A.; Storch, A.; Dietrichs, E.; Pirtosek, Z.; Henriksen, T.; Horne, M.; Devos, D.; et al. Viewpoint and practical recommendations from a movement disorder specialist panel on objective measurement in the clinical management of Parkinson's disease. Npj Parkinsons Dis. 2018, 4, 14. [CrossRef]

7. Pahwa, R.; Isaacson, S.H.; Torres-Russotto, D.; Nahab, F.B.; Lynch, P.M.; Kotschet, K.E. Role of the Personal KinetiGraph in the routine clinical assessment of Parkinson's disease: Recommendations from an expert panel. Expert Rev. Neurother. 2018, 18, 669-680. [CrossRef] [PubMed]

8. Thorp, J.E.; Adamczyk, P.G.; Ploeg, H.L.; Pickett, K.A. Monitoring Motor Symptoms During Activities of Daily Living in Individuals With Parkinson's Disease. Front. Neurol. 2018, 9, 1036. [CrossRef] [PubMed]

9. Powers, R.; Etezadi-Amoli, M.; Arnold, E.M.; Kianian, S.; Mance, I.; Gibiansky, M.; Trietsch, D.; Alvarado, A.S.; Kretlow, J.D.; Herrington, T.M.; et al. Smartwatch inertial sensors continuously monitor real-world motor fluctuations in Parkinson's disease. Sci. Transl. Med. 2021, 13, e7865. [CrossRef]

10. Choi, K.W.; Chen, C.Y.; Stein, M.B.; Klimentidis, Y.C.; Wang, M.J.; Koenen, K.C.; Smoller, J.W. Assessment of Bidirectional Relationships Between Physical Activity and Depression Among Adults: A 2-Sample Mendelian Randomization Study. JAMA Psychiatry 2019, 76, 399-408. [CrossRef]

11. Heijmans, M.; Habets, J.G.V.; Herff, C.; Aarts, J.; Stevens, A.; Kuijf, M.L.; Kubben, P.L. Monitoring Parkinson's disease symptoms during daily life: A feasibility study. Npj Parkinsons Dis. 2019, 5, 21. [CrossRef]

12. Vizcarra, J.A.; Sanchez-Ferro, A.; Maetzler, W.; Marsili, L.; Zavala, L.; Lang, A.E.; Martinez-Martin, P.; Mestre, T.A.; Reilmann, R.; Hausdorff, J.M.; et al. The Parkinson's disease e-diary: Developing a clinical and research tool for the digital age. Mov. Disord. 2019, 34, 676-681. [CrossRef] [PubMed]

13. Neurotechnologies, G.L. Kinesia ProViewTM. Available online: https://www.glneurotech.com/blog/2013/05/15/press-releasekinesia-proview-launches-to-visualize-parkinsons-symptom-response-to-deep-brain-stimulation-programming/ (accessed on 20 November 2017).

14. Rodriguez-Molinero, A.; Perez-Lopez, C.; Sama, A.; de Mingo, E.; Rodriguez-Martin, D.; Hernandez-Vara, J.; Bayes, A.; Moral, A.; Alvarez, R.; Perez-Martinez, D.A.; et al. A Kinematic Sensor and Algorithm to Detect Motor Fluctuations in Parkinson Disease: Validation Study under Real Conditions of Use. JMIR Rehabil. Assist. Technol. 2018, 5, e8. [CrossRef] [PubMed]

15. Warmerdam, E.; Hausdorff, J.M.; Atrsaei, A.; Zhou, Y.; Mirelman, A.; Aminian, K.; Espay, A.J.; Hansen, C.; Evers, L.J.; Keller, A. Long-term unsupervised mobility assessment in movement disorders. Lancet Neurol. 2020, 19, 462-470. [CrossRef]

16. Fasano, A.; Mancini, M. Wearable-based mobility monitoring: The long road ahead. Lancet Neurol. 2020, 19, 378-379. [CrossRef] 
17. Kelly, C.J.; Karthikesalingam, A.; Suleyman, M.; Corrado, G.; King, D. Key challenges for delivering clinical impact with artificial intelligence. BMC Med. 2019, 17, 195. [CrossRef]

18. Pencina, M.J.; Goldstein, B.A.; D'Agostino, R.B. Prediction Models-Development, Evaluation, and Clinical Application. N. Engl. J. Med. 2020, 382, 1583-1586. [CrossRef]

19. Kluge, F.; Del Din, S.; Cereatti, A.; Gaßner, H.; Hansen, C.; Helbostadt, J.L.; Klucken, J.; Küderle, A.; Müller, A.; Rochester, L.; et al. Consensus based framework for digital mobility monitoring. medRxiv 2020. [CrossRef]

20. Galperin, I.; Hillel, I.; Del Din, S.; Bekkers, E.M.J.; Nieuwboer, A.; Abbruzzese, G.; Avanzino, L.; Nieuwhof, F.; Bloem, B.R.; Rochester, L.; et al. Associations between daily-living physical activity and laboratory-based assessments of motor severity in patients with falls and Parkinson's disease. Parkinsonism Relat. Disord. 2019, 62, 85-90. [CrossRef]

21. Goetz, C.G.; Tilley, B.C.; Shaftman, S.R.; Stebbins, G.T.; Fahn, S.; Martinez-Martin, P.; Poewe, W.; Sampaio, C.; Stern, M.B.; Dodel, R.; et al. Movement Disorder Society-sponsored revision of the Unified Parkinson's Disease Rating Scale (MDS-UPDRS): Scale presentation and clinimetric testing results. Mov. Disord. 2008, 23, 2129-2170. [CrossRef]

22. Hagell, P.; Nygren, C. The 39 item Parkinson's disease questionnaire (PDQ-39) revisited: Implications for evidence based medicine. J. Neurol. Neurosurg. Psychiatry 2007, 78, 1191-1198. [CrossRef] [PubMed]

23. Evers, L.J.W.; Krijthe, J.H.; Meinders, M.J.; Bloem, B.R.; Heskes, T.M. Measuring Parkinson's disease over time: The real-world within-subject reliability of the MDS-UPDRS. Mov. Disord. 2019, 34, 1480-1487. [CrossRef]

24. Heijmans, M.; Habets, J.; Kuijf, M.; Kubben, P.; Herff, C. Evaluation of Parkinson's disease at home: Predicting tremor from wearable sensors. In Proceedings of the 41st Annual International Conference of the IEEE Engineering in Medicine and Biology Society (EMBC), Berlin, Germany, 23-27 July 2019; pp. 584-587.

25. Todorova, A.; Jenner, P.; Ray Chaudhuri, K. Non-motor Parkinson's: Integral to motor Parkinson's, yet often neglected. Pract. Neurol. 2014, 14, 310. [CrossRef]

26. Seppala, J.; De Vita, I.; Jamsa, T.; Miettunen, J.; Isohanni, M.; Rubinstein, K.; Feldman, Y.; Grasa, E.; Corripio, I.; Berdun, J.; et al. Mobile Phone and Wearable Sensor-Based mHealth Approaches for Psychiatric Disorders and Symptoms: Systematic Review. JMIR Ment. Health 2019, 6, e9819. [CrossRef] [PubMed]

27. Simons, C.J.; Hartmann, J.A.; Kramer, I.; Menne-Lothmann, C.; Hohn, P.; van Bemmel, A.L.; Myin-Germeys, I.; Delespaul, P.; van Os, J.; Wichers, M. Effects of momentary self-monitoring on empowerment in a randomized controlled trial in patients with depression. Eur. Psychiatry 2015, 30, 900-906. [CrossRef]

28. Broen, M.P.; Marsman, V.A.; Kuijf, M.L.; Van Oostenbrugge, R.J.; van Os, J.; Leentjens, A.F. Unraveling the Relationship between Motor Symptoms, Affective States and Contextual Factors in Parkinson's Disease: A Feasibility Study of the Experience Sampling Method. PLoS ONE 2016, 11, e0151195. [CrossRef]

29. Mahadevan, N.; Demanuele, C.; Zhang, H.; Volfson, D.; Ho, B.; Erb, M.K.; Patel, S. Development of digital biomarkers for resting tremor and bradykinesia using a wrist-worn wearable device. Npj Digit. Med. 2020, 3, 5. [CrossRef] [PubMed]

30. Rodriguez-Molinero, A. Monitoring of Mobility of Parkinson's Patients for Therapeutic Purposes-Clinical Trial (MoMoPa-EC). Available online: https:/ / clinicaltrials.gov/ct2/show /NCT04176302 (accessed on 18 November 2020).

31. Michael, J. Fox Fundation. Beat-PD Dream Challenge (by Sage Bionetworks; Michael, J. Fox Foundation). Available online: https: / / www.michaeljfox.org/grant/beat-pd-biomarker-and-endpoint-assessment-track-parkinsons-disease-dream-challenge (accessed on 7 January 2021).

32. Loring, D.W.; Block, C.; Staikova, E.; Miocinovic, S. Patient-Reported Outcomes Measurement Information System (PROMIS) Assessment of Non-Motor Features in Deep Brain Stimulation Candidates: Relationship to the Beck Depression and Anxiety Inventories. Arch. Clin. Neuropsychol. 2020. [CrossRef]

33. Bloem, B.R.; Marks, W.J.J.; Silva de Lima, A.L.; Kuijf, M.L.; van Laar, T.; Jacobs, B.P.F.; Verbeek, M.M.; Helmich, R.C.; van de Warrenburg, B.P.; Evers, L.J.W.; et al. The Personalized Parkinson Project: Examining disease progression through broad biomarkers in early Parkinson's disease. BMC Neurol. 2019, 19, 160. [CrossRef]

34. Rochester, L.; Mazzà, C.; Mueller, A.; Caulfield, B.; McCarthy, M.; Becker, C.; Miller, R.; Piraino, P.; Viceconti, M.; Dartee, W.P.; et al. A Roadmap to Inform Development, Validation and Approval of Digital Mobility Outcomes: The Mobilise-D Approach. Digit. Biomark. 2020, 4, 13-27. [CrossRef]

35. Habets, J.; Kubben, P. EMA and Wearable Sensor Monitoring in PD, V2 ed.; Maastricht University: Maastricht, The Netherlands, 2020. [CrossRef]

36. Kemp, B.; Varri, A.; Rosa, A.C.; Nielsen, K.D.; Gade, J. A simple format for exchange of digitized polygraphic recordings. Electroencephalogr. Clin Neurophysiol. 1992, 82, 391-393. [CrossRef]

37. Verhagen, S.J.; Hasmi, L.; Drukker, M.; van Os, J.; Delespaul, P.A. Use of the experience sampling method in the context of clinical trials. Evid. Based Ment. Health 2016, 19, 86-89. [CrossRef] [PubMed]

38. Brannon, E.E.; Cushing, C.C.; Crick, C.J.; Mitchell, T.B. The promise of wearable sensors and ecological momentary assessment measures for dynamical systems modeling in adolescents: A feasibility and acceptability study. Transl. Behav. Med. 2016, 6, 558-565. [CrossRef] [PubMed]

39. Palmier-Claus, J.E.; Myin-Germeys, I.; Barkus, E.; Bentley, L.; Udachina, A.; Delespaul, P.A.; Lewis, S.W.; Dunn, G. Experience sampling research in individuals with mental illness: Reflections and guidance. Acta Psychiatr. Scand. 2011, 123, 12-20. [CrossRef] 
40. Habets, J.; Heijmans, M.; Herff, C.; Simons, C.; Leentjens, A.F.; Temel, Y.; Kuijf, M.; Kubben, P. Mobile Health Daily Life Monitoring for Parkinson Disease: Development and Validation of Ecological Momentary Assessments. JMIR mHealth uHealth 2020, 8, e15628. [CrossRef]

41. Papapetropoulos, S.S. Patient diaries as a clinical endpoint in Parkinson's disease clinical trials. CNS Neurosci. Ther. 2012, 18, 380-387. [CrossRef] [PubMed]

42. Erb, M.K.; Karlin, D.R.; Ho, B.K.; Thomas, K.C.; Parisi, F.; Vergara-Diaz, G.P.; Daneault, J.F.; Wacnik, P.W.; Zhang, H.; Kangarloo, T.; et al. mHealth and wearable technology should replace motor diaries to track motor fluctuations in Parkinson's disease. Npj Digit. Med. 2020, 3, 6. [CrossRef]

43. Reback, J.; McKinney, W.; jbrockmendel; Van den Bossche, J.; Augspurger, T.; Cloud, P.; gfyoung; Sinhrks; Hawkins, S.; Klein, A.; et al. Psifr: Analysis and Visualization of Free Recall Data. Pandas-dev/pandas: Pandas 1.1.1, 2020 (Version v1.1.1) Zenodo. Available online: http:/ / doi.org/10.5281/zenodo.3993412 (accessed on 20 August 2020).

44. Van der Walt, S.; Colbert, S.C.; Varoquaux, G. The NumPy Array: A Structure for Efficient Numerical Computation. Comput. Sci. Eng. 2011, 13, 22-30. [CrossRef]

45. Pedregosa, F.; Varoquaux, G.; Gramfort, A.; Michel, V.; Thirion, B.; Grisel, O.; Blondel, M.; Prettenhofer, P.; Weiss, R.; Dubourg, V. Scikit-learn: Machine learning in Python. J. Mach. Learn. Res. 2011, 12, 2825-2830. 